\title{
Patient with Down Syndrome and relapsed acute lymphoblastic leukemia with sustained remission despite only partial British R3 chemotherapy
}

\author{
Zhongbo $\mathrm{Hu}^{1}$, Kristen VanHeyst ${ }^{1}$, jignesh dalal ${ }^{1}$, and Lisa Hackney ${ }^{1}$ \\ ${ }^{1}$ UH Rainbow Babies and Children's Hospital
}

May 11, 2020

\begin{abstract}
Relapsed acute lymphoblastic leukemia (ALL) with high minimal residual disease (MRD) after reinduction has poor prognosis. ALL patients with Down syndrome (DS) have a higher rate of relapse and treatment related mortality. We describe a DS and ALL patient with late bone marrow relapse who was treated per British ALL R3 reinduction chemotherapy. His therapy was discontinued due to severe systemic infections. The patient remained in remission for approximately two years without further treatment. We hypothesize that the sustained remission is attributed to chemotherapy combined with increased cortisol production and cytokine release as a result of systemic stress from infections.
\end{abstract}

\section{INTRODUCTION}

The current treatment regimens for acute lymphoblastic leukemia have successfully attained cure rates of approximately $90-95 \%^{1,2}$. A remaining significant challenge, however, is to improve the survival of patients with relapsed ALL. Patients with relapsed ALL have a suboptimal prognosis ${ }^{1,3}$. Down syndrome patients with relapsed ALL have even worse outcomes. DS itself was proved to be an independent prognostic factor of outcome after ALL relapse. ${ }^{4}$ There are several case reports of patients with leukemia who attained spontaneous remission after severe infection ${ }^{5-7}$. There are no reports of a patient with relapsed leukemia with sustained remission following cessation of therapy secondary to severe infections, except an older patient with relapsed FLT3 internal tandem duplication mutant acute myeloid leukemia whose disease underwent spontaneous remission for 35 days without precipitating cause ${ }^{8}$.

We describe a case of a 37 year-old man with Down syndrome and ALL who had a late bone marrow relapse and sustained remission for 2 years after only receiving three cycles of ALL R3 chemotherapy due to severe systemic infections.

\section{CASE REPORT}

A 30 year-old male with Down syndrome presented with fever, cough, and shortness of breath in November 2013. His complete blood count (CBC) showed pancytopenia with a white blood cell (WBC) count of 1.4 x $10^{\wedge} 9 / \mathrm{L}$, hemoglobin of $8.3 \mathrm{~g} / \mathrm{dL}$, and platelet count of $32 \times 10^{\wedge} 9 / \mathrm{L}$ with occasional circulating blasts. Bone marrow aspirate and biopsy confirmed the diagnosis of acute lymphoblastic leukemia with $73 \%$ blasts which co-expressed CD34, CD19, CD10 and TdT with aberrant expression of dim CD33 by flow cytometry, which was consistent with B-precursor ALL. Cytogenetic analysis was not successful but FISH from a bone marrow sample obtained on day 17 during Induction chemotherapy as per the Children's Oncology Group (COG) study AALL1131 showed nuc ish(ETV6x2,RUNX1x3)[250] which was a normal signal pattern for constitutional trisomy 21 with two ETV6 signals and three RUNX1 signals. Cerebrospinal fluid was negative for blasts. 
The patient was treated per COG high risk protocol AALL1131, Down syndrome arm due to NCI risk factor for age above 10 years old. He had a rapid early response with a M1 marrow at Day 17 and he achieved complete remission after Induction with negative minimal residual disease (MRD). He had a complicated treatment course including a few episodes of sepsis, one of which included multi-organ failure for which he was in the Medical ICU, and PICC line associated DVT, Following the end of his treatment, his platelet count never normalized and ranged between $90 \times 10^{\wedge} 9 / \mathrm{L}$ and $112 \times 10^{\wedge} 9 / \mathrm{L}$. At his lowest level of thrombocytopenia to $48 \times 10^{\wedge} 9 / \mathrm{L}$ in September 2017, a repeat bone marrow aspirate and biopsy was obtained and showed a $90 \%$ cellular bone marrow with $82 \%$ blasts, consistent with bone marrow relapse. FISH showed CRLF2 translocation positive in $63.5 \%$ of cells and was also positive for deletion of CDKN2A on chromosome $9 \mathrm{p}$ beside his constitutional trisomy of chromosome 21. CNS was negative. This relapse was treated with Reinduction chemotherapy per ALL R3 protocol. Post-reinduction day 29 MRD was $0.2 \%$ (Table 1).

He continued to receive Consolidation chemotherapy per ALL R3 protocol, after which his bone marrow had negative MRD. While hospitalized for Intensification chemotherapy, he developed sepsis, necrotizing pneumonia, and severe bilateral pleural effusions. BAL revealed Candida albicans. CT imaging also revealed near complete splenic infarct, right renal perfusion deficits with signs of liquefaction and a segmental area of abnormal small bowel wall enhancement and concern for focal small bowel wall defect. He also developed delirium related to his severe illness and prolonged hospitalization. Due to severe illness, his chemotherapy was held during a three month period. A bone marrow after three months continued to demonstrate negative MRD. Once he became more clinically stable, chemotherapy was attempted with intermediate dosed Methotrexate but this hospital course was complicated by infection necessitating removal of his broviac, fluid overload, and a persistent cavitary lung lesion. At this point, a decision was made by his family and treatment team to stop chemotherapy. About 50 weeks after his diagnosis of relapsed disease, a repeat bone marrow continued to demonstrate negative MRD. He was followed closely as an outpatient. Unfortunately, he presented with acute onset psychosis and thrombocytopenia about 2 years after stopping his treatment and bone marrow was consistent with relapsed disease. Family opted for no further therapy and unfortunately the patient passed away 7 weeks later.

\section{DISCUSSION}

Despite current cure rates of ALL above $90 \%{ }^{1,9}$, relapsed ALL is still the most important obstacle for the cure of pediatric ALL patients. The relapse rate is about 10-15\% of ALL in children, but substantially higher (about 25-30\%) in high-risk subgroups ${ }^{10,11}$. An estimated $40 \%$ to $50 \%$ of adult patients with ALL experience relapse ${ }^{12}$. Although high dose Mitoxantrone plus Cytarabine were effective in achieving remission in refractory leukemia, the duration of the remission was only about half a year ${ }^{13}$ and still about $50 \%$ of ALL patients do not respond to salvage therapy prior to the ALL R3 protocol ${ }^{14}$. The British ALL R3 protocol utilizes Mitoxantrone to confer a significant response in progression-free and overall survival in children with relapsed ALL ${ }^{3}$. Our patient, who had a bone marrow relapse about 20 months after the completion of his initial chemotherapy, was considered to have late bone marrow relapse (defined as relapse occurring 36 months after the first diagnosis or more than 6 months after the end of front-line therapy). He was treated as per the ALL R3 protocol and had positive MRD after Reinduction and negative MRD after Consolidation. Per the ALL R3 protocol, patients with B-precursor ALL with late bone marrow relapse and low MRD $(<0.01 \%)$ at the end of induction had favorable outcomes with chemotherapy without undergoing stem-cell transplantation. Patients with higher MRD $(>0.01 \%)$ required allogeneic stem-cell transplantation. But after long-term follow-up of the late bone marrow relapse patients for 7 years, the second relapse rate was about $23 \%$ in the high MRD group even after stem cell transplant. In the low MRD group with chemotherapy, second relapse rate was about $27 \%^{15}$. The progression-free survival at 5 years was significantly lower in the high MRD group comparing with that in the low MRD with $56 \%$ versus $72 \%^{15}$. Our case is unique in that the patient remained in remission for nearly two years after only receiving three phases of chemotherapy per the ALL R3 protocol for his first relapse.

Children with Down syndrome are well-known to have increased risk of developing B-cell precursor ALL. The Ponte di Legno study group showed that DS-ALL patients had higher 8-year cumulative incidence 
of relapse than non-DS patients (26\% vs $15 \%)^{16}$. Treatment-related mortality, primarily from infection, is increased in all protocols in DS-ALL, which made both the five-year event-free and overall survival inferior in patients with DS-ALL ${ }^{17,18}$. Current COG protocols have made modifications to limit toxicities. Our patient had multiple infections during his initial chemotherapy per AALL1131. He also developed multiple severe infections after three phases of chemotherapy per the ALL R3 protocol following disease relapse. Given the significant toxicity that he experienced, a decision was made for cessation of therapy. Over time, more options are becoming available for patients with relapsed ALL that confer less toxicity. Currently, antibody-targeted therapies, such as bispecific (CD3/CD19) T-cell-engaging antibody Blinatumomab, Inotzumab, and CD19directed chimeric antigen receptor $(\mathrm{CAR}) \mathrm{T}$-cell therapies are major breakthroughs in the management of relapsed leukemia ${ }^{19}$.

Spontaneous or transient remission of acute lymphoblastic leukemia during severe infection has been described in a few cases and case series reports with averaged ten weeks in duration ${ }^{5-7,20}$. The proposed mechanism was due to increased cortisol production during stress or infection combined with an immunemediated process thereby generating anti-leukemic effects ${ }^{5,6,21}$. Cytokines, including tumor necrosis factoralpha, interferon-alpha, and interleukin-2, released during infection can directly inhibit the residual blast proliferation or through increased activity of $\mathrm{T}$ lymphocytes, macrophages, and natural killer cells leading to an anti-leukemia effect ${ }^{22,23}$. Similar phenomena and mechanisms have been discussed and reported in several cases and case series in acute myeloid leukemia patients ${ }^{24,25}$. Our patient's long-term remission may be attributed to a combination of the three phases of chemotherapy per ALL R3 protocol with the systemic stress related to his multiple severe infections.

\section{CONFLICTS OF INTEREST}

The authors declare no conflicts of interest.

1 Hunger, S. P. \& Mullighan, C. G. Acute Lymphoblastic Leukemia in Children. $N$ Engl J Med 373 , 1541-1552, doi:10.1056/NEJMra1400972 (2015).

2 Cooper, S. L. \& Brown, P. A. Treatment of pediatric acute lymphoblastic leukemia. Pediatr Clin North Am 62 , 61-73, doi:10.1016/j.pcl.2014.09.006 (2015).

3 Parker, C. et al. Effect of mitoxantrone on outcome of children with first relapse of acute lymphoblastic leukaemia (ALL R3): an open-label randomised trial. Lancet 376 , 2009-2017, doi:10.1016/S01406736(10)62002-8 (2010).

4 Meyr, F. et al. Outcomes of treatment for relapsed acute lymphoblastic leukaemia in children with Down syndrome. Br J Haematol 162, 98-106, doi:10.1111/bjh.12348 (2013).

5 Iqbal, A., Weinstein, J., Angelova, V., Dighe, D. \& Giordano, L. A Rare Case of Spontaneous Remission of Terminal Deoxynucleotidyl Transferase Negative B-acute Lymphoblastic Leukemia. J Pediatr Hematol Oncol 40 , e176-e178, doi:10.1097/MPH.0000000000000906 (2018).

6 Yoruk, A. et al. Spontaneous remission of acute lymphoblastic leukemia with mediastinal mass. Pediatr Hematol Oncol25 , 181-186, doi:10.1080/08880010801938132 (2008).

7 Hores, T., Wendelin, K. \& Schaefer-Eckart, K. Spontaneous remission of acute lymphoblastic leukemia: A case report. Oncol Lett15 , 115-120, doi:10.3892/ol.2017.7288 (2018).

8 Vachhani, P. et al. Spontaneous Remission in an Older Patient with Relapsed FLT3 ITD Mutant AML. Case Rep Hematol2016 , 1259759, doi:10.1155/2016/1259759 (2016).

9 Pal, S. K. et al. Clinical Cancer Advances 2019: Annual Report on Progress Against Cancer From the American Society of Clinical Oncology. J Clin Oncol 37 , 834-849, doi:10.1200/JCO.18.02037 (2019).

10 Teachey, D. T. \& Hunger, S. P. Predicting relapse risk in childhood acute lymphoblastic leukaemia. $\mathrm{Br}$ J Haematol 162, 606-620, doi:10.1111/bjh.12442 (2013). 
11 Bhojwani, D. \& Pui, C. H. Relapsed childhood acute lymphoblastic leukaemia. Lancet Oncol 14, e205217, doi:10.1016/S1470-2045(12)70580-6 (2013).

12 Jabbour, E., Pui, C. H. \& Kantarjian, H. Progress and Innovations in the Management of Adult Acute Lymphoblastic Leukemia. JAMA Oncol4, 1413-1420, doi:10.1001/jamaoncol.2018.1915 (2018).

13 Raanani, P. et al. Salvage therapy of refractory and relapsed acute leukemia with high dose mitoxantrone and high dose cytarabine.Leuk Res 23 , 695-700, doi:10.1016/s0145-2126(99)00078-8 (1999).

14 Fuster, J. Current approach to relapsed acute lymphoblastic leukemia in children. World J Hematol 3 , 49-70, doi:10.5315/wjh.v3.i3.49 (2014).

15 Parker, C. et al. Outcomes of patients with childhood B-cell precursor acute lymphoblastic leukaemia with late bone marrow relapses: long-term follow-up of the ALLR3 open-label randomised trial.Lancet Haematol 6 , e204-e216, doi:10.1016/S2352-3026(19)30003-1 (2019).

16 Buitenkamp, T. D. et al. Acute lymphoblastic leukemia in children with Down syndrome: a retrospective analysis from the Ponte di Legno study group. Blood 123, 70-77, doi:10.1182/blood-2013-06-509463 (2014).

17 Maloney, K. W. et al. Down syndrome childhood acute lymphoblastic leukemia has a unique spectrum of sentinel cytogenetic lesions that influences treatment outcome: a report from the Children's Oncology Group. Blood 116 , 1045-1050, doi:10.1182/blood-2009-07-235291 (2010).

18 Whitlock, J. A. Down syndrome and acute lymphoblastic leukaemia.Br J Haematol 135 , 595-602, doi:10.1111/j.1365-2141.2006.06337.x (2006).

19 Paul, S., Rausch, C. R., Nasnas, P. E., Kantarjian, H. \& Jabbour, E. J. Treatment of relapsed/refractory acute lymphoblastic leukemia. Clin Adv Hematol Oncol 17, 166-175 (2019).

20 Bierman, H. R. et al. Remissions in leukemia of childhood following acute infectious disease: staphylococcus and streptococcus, varicella, and feline panleukopenia. Cancer 6 , 591-605, doi:10.1002/10970142(195305)6:3<591::aid-cncr2820060317>3.0.co;2-m (1953).

21 Lynggaard, L. S., Marquart, H. V., Kjeldsen, E., Madsen, H. O. \& Hasle, H. Acute Lymphoblastic Leukemia Presenting With Pancytopenia Followed by a 14-Month-Long Period of Transient Remission Possibly Supporting the Adrenal Hypothesis of Leukemogenesis. J Pediatr Hematol Oncol 38, e271-e273, doi:10.1097/MPH.0000000000000648 (2016).

22 Jimemez, C. et al. Increased serum tumour necrosis factor during transient remission in acute leukaemia. Lancet341 , 1600, doi:10.1016/0140-6736(93)90739-4 (1993).

23 Musto, P. et al. Spontaneous remission in acute myeloid leukaemia: a role for endogenous production of tumour necrosis factor and interleukin-2? Br J Haematol 87, 879-880, doi:10.1111/j.1365-2141.1994.tb06761.x (1994).

24 Helbig, D. et al. Spontaneous Remission in a Patient With Acute Myeloid Leukemia Leading to Undetectable Minimal Residual Disease.J Hematol 9 , 18-22, doi:10.14740/jh606 (2020).

25 Rashidi, A. \& Fisher, S. I. Spontaneous remission of acute myeloid leukemia. Leuk Lymphoma $\mathbf{5 6}$, 1727-1734, doi:10.3109/10428194.2014.970545 (2015).

\section{Hosted file}

Table.docx available at https://authorea.com/users/320378/articles/449972-patient-with-downsyndrome-and-relapsed-acute-lymphoblastic-leukemia-with-sustained-remission-despiteonly-partial-british-r3-chemotherapy 